\title{
A Human-in-the-Loop Cyber-Physical Approach for Students Performance Assessment
}

\author{
J.Fernandes*, D.Raposo*, S.Sinche ${ }^{* \dagger}$, N.Armando*‡, J.Sa Silva*, A.Rodrigues*\#, L.Macedo*, \\ H.Gonçalo Oliveira*, F.Boavida* \\ ${ }^{*}$ CISUC, University of Coimbra, Coimbra Portugal \\ †DETRI-Escuela Politécnica Nacional, Quito Ecuador \\ ¥ESPU, Universidade Kimpa Vita, Uíge Angola \\ \# ISCAC, Politécnico Coimbra, Coimbra Portugal \\ +351938783035jmfernandes@dei.uc.pt
}

\begin{abstract}
As the number of smart devices that surround us increases, so do the possibilities of leveraging them to create intelligent, socially-aware systems. However, apart from e-health systems, few applications consider humans as active system players. In fact, most applications/systems have the sole objective of providing some service to humans without considering their intents, actions or emotions. In this respect, they can be looked at as open loop. Systems that do consider human feedback are called Human-in-the-Loop CyberPhysical-Systems (HiTLCPS), and integrate the humans in a feedback loop, with potential to react and change the environment. In this paper, we propose an approach that implements the HITLCPS concept. The developed platform, ISABELA, explores both online social networks (OSN) data as well as a variety of other data, including environment data and data from personal mobile devices, thus combining IoT, social sensors and mobile sensing. In addition to present the system architecture, the paper provides preliminary results obtained during field trials.
\end{abstract}

\section{CCS Concepts}

-Human-centered computing $\rightarrow$ Ubiquitous and mobile computing systems and tools; Empirical studies in ubiquitous and mobile computing;

\section{Keywords}

HiTLCPS, Smartphone Sensing, Social Sensors, IoT, Online Social Networks

\section{INTRODUCTION}

The term "Internet of Things" changed since it was first proposed, in 1999[1]. It now refers to the possibility to empower computers with means to gather data by themselves, so they can perceive the world without human in-

Permission to make digital or hard copies of all or part of this work for personal or classroom use is granted without fee provided that copies are not made or distributed for profit or commercial advantage and that copies bear this notice and the full citation on the first page. Copyrights for components of this work owned by others than ACM must be honored. Abstracting with credit is permitted. To copy otherwise, or republish, to post on servers or to redistribute to lists, requires prior specific permission and/or a fee. Request permissions from permissions@ acm.org.

SocialSense'19, April 15, 2019, Montreal, QC, Canada

(C) 2019 ACM. ISBN 978-1-4503-6706-6/19/04 . . \$15.00

DOI: https://doi.org/10.1145/3313294.3313387 put. Nowadays, the Internet of Things (IoT) is more than just a concept. Everyday devices are endowed with sensors and are connected to the Internet, allowing them to share and receive data. At the same time, these devices now have enough processing power to create new Cyber-Physical Systems (CPS) that can manage and control physical phenomena[2]. Moreover, we can apply these technologies to human-centered applications, where humans are the main component of control loops, thus leading to HiTLCPS[3]. In HiTLCPS, technology is driven by humans and considers human intents, actions (present and foreseen), emotions, and psychological states, thus creating systems that better care for human needs[4].

HiTLCPS enable an Internet that conveys emotions, psychological states, actions and drives humans as part of larger scale systems[4]. Hence, both sensing and actuation approaches go beyond physical electronic-based devices. Indeed, they also comprise software-based entities like artificial agents and human beings, by considering all humanoriginated activities from social media, for instance, as raw data. Like the embedded chips in electronic-based sensors, "human-based sensors" also need a mechanism to transform collected raw data into values that can be interpreted, thus conducting to information and knowledge[5]. Though, solutions in the community have not yet successfully joined HiTL and online social media (OSN)[4]. The attempt to overcome the aforementioned challenge is also one of the contributions of this paper.

Advances in the technology have also made it possible for almost every human to carry a computer in the palm of their hand or in their pocket. Nowadays smartphones have a processing power and memory that surpass by far the capacity of computers that in the past occupied an entire room. Furthermore, even the most modest smartphones are empowered with sensors and cameras. Smartphones have the necessary capabilities to run complex calculations, store data and have communication capabilities, which allows them to share sensed information. This provides a new way to look at human lives in a non-obtrusive way. We can now monitor humans in almost every situation as they now carry these portable computers with them to every place.

In this paper, we present our HiTLCPS called IoT Student Advisor and Best Lifestyle Analyzer (ISABELA). We developed a novel model to assess students' performances in a non-obtrusive and passive manner, by using smartphones, smartwatches, IoT and social sensors as sensing mechanisms. 
The contributions of this work are as follows: Use of OpenSource IoT platforms, technologies, standards and Interactive Agents to implement a new HiTLCPS system; Use of social networks as source of context-aware data; A dataset from a 30-days trial; Correlation of students' activity and self-reported data with their performance.

The rest of this article is organized as follows: Section II is a review of some of the state-of-the-art applications that incorporate or try to incorporate the HiTLCPS; Section III presents our case study, namely its environment, architecture, the Android application and its sensors (physical, virtual and the social); Section IV reports and discusses some results of the platform use, including in some of the mentioned trials. Conclusions and guidelines for further work are provided in section $\mathrm{V}$.

\section{RELATED WORK}

As stated before, not many works fully implement the HiTLCPS model, but a few works come close to it. Also, in the past, numerous works have presented Human-centered applications (e.g. $[6,7])$ and as such fall upon the HiTLCPS domain as well. In this section, we present an overview of some works that were developed as or proposed an HiTLCPS concept. Lane N, et. al., 2011[8] developed "BeWell", a system that measured physical activity, sociability and sleep throw the smartphone sensors, namely GPS, accelerometer and microphone. In this study, they make a close loop approach where they monitor the people's day-to-day activities passively, infer their well-being and inform them about the assessment made. The authors developed two applications to give information to the users, first through a widget in the smartphone where they showed to the users their stats on the sleep, activity and sociability measures. And the second, a web portal where the user could see more information. However, they only gave feedback with scores for the physical activity, social interactions and sleep. Although this is still valuable information that the humans could use to improve their well-being, this work could have been extended by performing a more context-aware actuation, and by giving information that is tailored for that specific person.

The previous work was extended[9] with a study named "StudentLife". Its goal was to correlate students' academic performance with psychological and physical well-being, using a mobile application and self-reports. Using their system, they conducted a study in Dartmouth's University, where a class of 48 students was monitored during a 10week period. Some of the results presented on that study were: a significant inverse correlation between sleep duration and pre and post-depression. The same was found to be true for conversations frequency (students that have less conversations are more likely to be depressed) and overall social activity (students that are more social and are surrounded by people are less likely to get depressed). Yet, no feedback mechanism was implemented or proposed to give awareness to the students.

Other authors also exploited the use of IoT devices and wearables in order to monitor humans, as in "HappyOffice"[10]. The main objective of that study was the detection of people's mood in the workplace. For this task they used 3 types of devices, 2 variants of the Toshiba SilmeeTM (Bar Type and Wristband) and the smartphone as a mean to obtain the ground-truth. The Toshiba devices were able to collect physiological data (namely the heart rate, the pulse rate and skin temperature) and 3-axial acceleration. The ground-truth was obtained through a self-reporting app. They also conducted a real study where 4 researchers, working at the same office, were monitored during 11 working days. A framework was also proposed for incorporating all the phases of the HiTLCPS concept. The framework proposal consisted of the work presented plus an additional actuation phase where individual feedback is given to the workers, in order to improve their mood. Furthermore, the authors also proposed an aggregation mechanism that would extract the aggregated mood for the office. This value could then be used by the employer as a mean to improve the overall quality of the mood in the office and improve the outcome of their workers.

In all the works reviewed, the same recurrent limitation was noted, when considering a HiTLCPS architecture: the lack of actuation or efficient actuation through contextualized feedback. Yet, some works propose that this phase of the HiTLCPS should be explored in their future work and some works give raw data to the users. The work in this field seems to be all on the efficiency of data acquisition and in the modelling of the human behavior, that are by themselves challenging. In our case study, we effectively tackle this issue.

\section{THE ISABELA SYSTEM}

The aim of ISABELA is to create a HiTLCPS capable of assisting users, by gathering physical data, behavioral data, and social networking data. Initially intended for assisting students in maximizing their academic performance, it can be used for any kind of user in a variety of scenarios. Nevertheless, in this paper, we focus on the student scenario.

\subsection{The HiTL Architecture}

ISABELA was built with a set of components from FIWARE [11], allowing for modularity and maximizing interoperability. FIWARE is a European project supported by the FI-PPP (Future Internet Public-Private Partnership) that aims to offer a set of open-source components, openstandards, and an open ecosystem to deal with some of the challenges of the new IoT-enabled Internet. The project developed the concept of Generic Enablers (GE), designed with well-defined Application Program Interfaces (APIs) targeting specific problems in the IoT domain. In the ISABELA project, we used several GEs from FIWARE, as depicted in Figure 1.

To deal with the sensing state, the IDAS GE[12][12] was used to manage all kinds of IoT devices. The component supports management and interoperability capabilities, between devices (that communicate using a variety of protocols) and the FIWARE platform. This GE allows the ISABELA boxes, built over Arduino and Raspberry Pi, to communicate with the FIWARE. These boxes are able to collect temperature, humidity, noise and luminosity data in the user environment. In the student scenario, these boxes were placed at school and at home.

HiTLCPS need to be scalable, so dynamic models are needed in order to represent the data of such systems. To have such dynamic ecosystem, the FIWARE project adopts the NGSI9/10 information model, previously developed by OMA[13]. The model is based on entities and attributes. Each entity has its own type and is represented by attributes using JSON. To improve the system distribution and scala- 


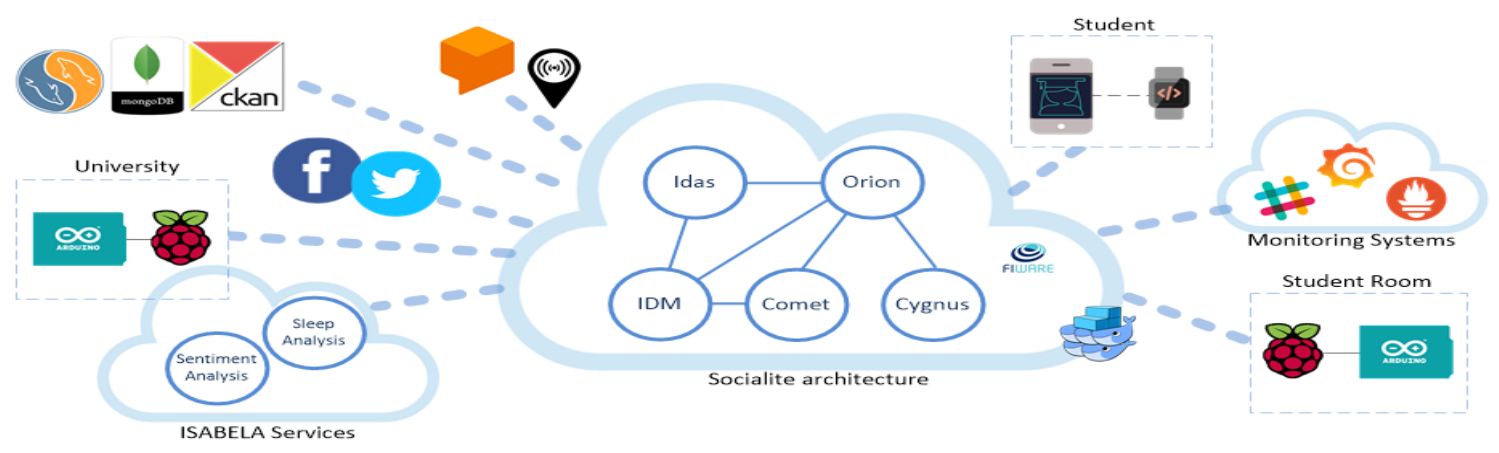

Figure 1: ISABELA HiTL system architecture.

bility, the module acts as a broker, by allowing external systems (consumers) to make data subscriptions with specific rules to the entities and attributes. ORION sends notifications to these systems when subscription rules are fired.

The ORION GE is not fit for storing historic data. Instead, the Cygnus[14] module is the connector "in charge of persisting certain sources of data" in several storages, using the Apache Flume technology and by subscribing to the ORION entities. Thus, when an entity is created/updated on ORION the Cygnus GE will trigger a specific channel and forward the data to a third-party data storage (e.g., MySQL, MongoDB and CKAN channel).

Databases usually do not provide APIs to retrieve the data to applications. Hence, another module of the FIWARE platform handles this issue, namely the Short-Term-History or STH-Comet[15] GE, which provides a RESTFUL API with historic-queries capabilities, and aggregated methods.

Security and privacy are some of the most important requirements of HiTLCPS systems. In order to protect communications between GEs, IoT devices and applications, the Identity Management Generic Enabler Keyrock, or IDM[16], was used. Keyrock allows adding authentication and security to devices, users, and applications, as well as authorization policies. Additionally, from a privacy point-of-view, and in order to meet all requirements from European privacy laws, all data handled by the Android application is anonymized.

ISABELA includes sleep analysis and sentiment analysis modules. The sleep analysis module is the component in charge of computing users' sleep hours, on the basis of data collected using smartphone sensors. The sentiment analysis module processes the text retrieved from both the users' Facebook and Twitter timelines and identifies transmitted sentiments.

Last but not least, the system provides feedback to the users through a chatbot, using a human-computer interaction technology based on natural language conversations, named Dialog Flow[17]. The ISABELA bot is capable of retrieving data from the set of APIs available in the ISABELA ecosystem, and subsequently provide user recommendations (see section 3.4).

\subsection{Data Acquisition}

The acquisition of data by the Smartphone is either made by direct acquisition of the sensor values, or by context acquisition, as we use one or more sensors to acquire a virtual sensor value (for example class attendance) or we get context information from the Smartphone's SMS and calls.

The physical sensors are implemented in the smartphone by hardware and their values can be obtained directly with an API that the Android SDK makes available. This API allows us to implement listeners to each sensor we want to monitor. Our application records the value of the sensor each 5 seconds and records them in a local database. That database is then emptied to the cloud when a network access is available. The physical sensors currently used by our application are as follows: proximity sensor, light sensor, accelerometer and gyroscope.

GPS can also be considered a physical sensor and we also acquire its data in our application. However, this required a different approach from the one explained before, since the GPS is a very battery consuming hardware. We used the Fused Location Provider API. After some usability tests, where we monitor the consumption of our smartphones' battery life during a day, we were able to define conditions for the GPS acquisition. Those conditions were: a minimal distance between updates of 10 meters, a minimal time between updates of 5 minutes, a maximum waiting time of 1 minute, a fastest interval for updates of 5 seconds, and a priority that balances the power and precision.

The microphone in the smartphone can also be perceived as a sensor. In our application, we use it to record the mean sound amplitude in the student's surroundings, each $30 \mathrm{~s}$. To address privacy issues, neither audio nor conversations are recorded. Furthermore, no audio files are stored on the smartphone nor in FIWARE.

Additionally, we infer physical Activity from the physical sensors. The Activity Recognition API provided by the Android SDK automatically detects activities by periodically reading short bursts of sensor data and processing them using machine learning models. The API is able to detect 8 activities, namely: in vehicle, on foot, running, walking, on bicycle, walking, tilting and still. However, we were only interested in detecting whether the user was active or not. As such, we grouped all the activities that represent exercise within one with the same name. Thus, running, on foot and on bicycle were assigned the value exercise in our dataset.

Apart from the physical sensors, we acquired context data from the smartphone. More specifically, we used one or more sensors and information stored in the smartphone to obtain context aware data. Information obtained this way includes: the calendar information, the SMS information, the calls information, the alarms information, discrete location information, indoor location and Social Networks' information. 
Once again, to address privacy, neither the SMS nor the calls information is stored, only the number of outgoing and incoming texts and calls is analyzed.

In our project, we also use Social Sensors. The idea behind this concept is to leverage the capability of humans in providing contextualized data via social media, which can be interpreted, conducting to information and knowledge[18]. Once a day, ISABELA processes the timeline posts from the user's social media account and sends it to FIWARE. When that information reaches the FIWARE-ORION, a subscription reroutes the information to the SA module. This module performs natural language processing in the data collected from social networks, which is our approach to interpret the posts, conducting to information and knowledge. This feature of our work will be more detailed in future publications[19].

All the data collected on the smartphone is mapped to an entity on ORION in our FIWARE deployment. We mapped this information with an id obtained by applying a one-way secure hash function to the users Facebook's account identification number. This makes very hard for someone to discover the data origin.

\subsection{Processing/Inference}

In this section, we present three data processing mechanisms: Activity, Location and Sleep data. Although we consider Sleeping as an activity, the sleeping data classification is processed in a different way from the activity classification and, as such, it deserves a more detailed explanation.

As stated on the previous section, we used the Google Activity Recognition API to infer user activity. Although this makes our work easier, it also limits our processing options. This API returns a list of possible activities and the confidence on each one (the reliability of that result). We consider the most probable activity, which is the one with the highest confidence, as the current user's activity. If none of the returned, activities have a confidence superior to 0.3 we consider the user activity as "Unknown", because, we couldn't classify it.

The discrete Location information is obtained through the Wi-Fi networks available. More precisely, we consider three possible locations: Home, University and Other. We get the available networks through a Wi-Fi scan that returns a list with the Service Set Identifier (SSID) and the strength of the signal for each network. We then check if any network corresponds to the network of the University (Eduroam network) or to the User's House network, initially picked by the user from the list of configured networks in the phone, at the time of the first Login.

This technique to determine discrete location, together with the GPS information, can give us a general sense of the students' whereabouts. However, there are networks that can be available in more than one location. The "Eduroam" network, for example, is available in all the University facilities and this makes our task of perceiving the student real location more difficult. To solve this issue, we also implemented an indoor tracking mechanism. For the indoor location, the FIND framework ${ }^{1}$ allows us to create a model based on Wi-Fi finger-printing. More precisely, we created a dataset with several samples of the Wi-Fi network in different points of a building and label them with a name to a specific location. We also used the location to check class

\footnotetext{
${ }^{1}$ FIND Framework: https://github.com/schollz/find
}

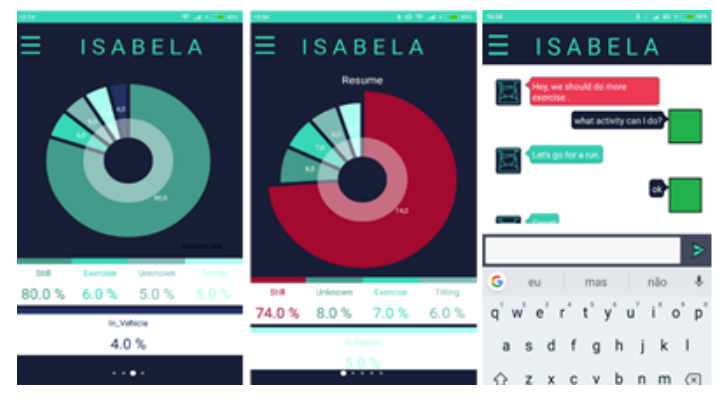

Figure 2: Normal screen(a); Visual clue for alarm(b); Chatbot alarm for low exercise(c).

attendance. By cross-referencing the user's schedule information with the indoor tracking mechanism result, we can determine if they are in fact attending the class.

In our study, we select seven features to perform sleep detection, namely: light intensity, phone lock state, day of the week, time to the next alarm, pressure sensor value, activity and sound amplitude. These seven features were selected based on the literature[6, 7]. The sleeping classifier training mechanism was too computationally demanding to run on an average smartphone, as such we choose to implement all the classification mechanism in our server-side application. Further details of this methodology will be addressed in future publications.

Apart from the Inference of states, the HiTLCPS concept also contemplates an inference of future states. However, in our system, that will only be implemented in future work, as it required us to first implement mechanisms for modelling the Students behavior.

\subsection{Actuation}

As part of the HiTLCPS concept we also implemented a mechanism of automatic feedback. Our primary objective in this case study is to improve the studentâĂ Źs academic performance through a control loop system. As such, we implemented two main methods of active feedback in our Android application, in addition to the passive information that the user can see in the main screens of the application, namely the visual clues and the interactive agent.

We have five types of alarms that will trigger the actuation: the user spends too much time still; the user has a lower exercise level during a week; the user spends little time in the university; the user spends too much time at home; or the user sleeps less than 6 hours.

The first type of actuation is feedback through visual clues in the mobile application. As depicted in Figure 2(b), when an alarm is raised, the display of the application changes. In case an alarm for lack of activity is lifted, the Still section of the graph will change to a red color and be highlighted. The same type of visual clue also happens to both the Sociability and Location charts.

The second method to perform actuation is through our implemented interactive agent, more commonly known as a chatbot. To implement the chatbot, we used the Dialogflow framework by Google ${ }^{2}$. In our project, we wanted not only to give feedback to the student, but also to contextualize said feedback. The use of an interactive agent allows us to

\footnotetext{
${ }^{2}$ Dialogflow: https://dialogflow.com/
} 
create a method in which each student receives personalized feedback. Additionally, the chatbot can answer follow up questions to the actuation, which makes the feedback more precise as well. This allowed us to quickly implement the chatbot infrastructure and focus on the development of the actuation itself.

The actuation of the chatbot works through the same alarm mechanism as the visual clues. More precisely, when an alarm is raised, the mobile application sends a notification with a message from the chatbot. By clicking it, the user is moved to the chatbot screen, where he can get more information about the alarm. As depicted in Figure 3 the alarm messages in the chatbot are also shown in red. If the user answers the notification within a 5-minute window, the chatbot retains the context and is able to give them more context. From the example of the Figure 2(c), if the chatbot gives an alarm for a lower exercise level, the user can then ask, "what activity can I do?" and the chatbot gives will give him a suggestion. Additionally, in the future, the chatbot will give feedback with the student location in mind or even by taking in account his schedule. For example, for some students that are near a park, the chatbot can suggest that location for exercising; or by detecting that the student still has classes, it can schedule that activity for later that day. This allows for a more context aware actuation, as the chatbot can interactively give more personalized feedback to each user. We also implemented some functionalities that make the chatbot more useful to the student. The chatbot is able to get context from the text the user writes and answer simple questions, such as giving information about the environment conditions retrieved from the IoT boxes that are part of the ISABELA architecture. The chatbot is also able to make simple tasks such as setting an alarm, showing the student classes, or the information about the available meals at the cafeteria.

\section{RESULTS}

ISABELA was subject to several tests, involving real users, more specifically, students in their academic context. This section provides some results, organized into 2 sections A) results of a trial whose objective was to correlate users (i.e., students) activity with their academic performance; and B) results that correlate the self-reported data with the students' performance. The two trials were conducted in Escuela Politécnica Nacional, in Ecuador, from the 12th of May to the 12 th of June 2018, with a total of 30 participating students. It is also worth mentioning that, in Ecuador, the first term goes from the 9th of April to the 8th of August. Thus, our study was in the middle of the first term and we were able to gather data during the mid-term exams. This is important, since, in order to obtain a positive classification, students need to have at least 12 points in the sum of the mid-term and the final exams, with both exams graded from 0 to 10 . As such, we can consider that any grade above 6 is positive, while any grade below 6 denotes poor performance from the student.

\subsection{Activity-performance correlation results}

In this section, we present some of the results extracted from the Ecuador's dataset, for the students' performance based on their activity. We divided the dataset into two groups, based on the student grades in the mid-term exam, group 1 (G1) comprising the students that obtained a grade of 6 or less, and group 2 (G2) comprising the remaining students. G1 had 12 students and G2 had 18 students.

As previously stated, we have 4 possible classifications for the activity detection: still, exercise, unknown or in vehicle. In this section, we present the results for comparing groups in each metric. It is also important to refer that, although we choose the label "Exercise", the classifications represent any continuous movement done by the user on foot, on bicycle or running. However, these movements need to be done with the smartphone in order to be recorded. As such, this data is not representative of moments in which the user chooses to exercise without the smartphone (e.g. going to the gym). Nonetheless this data can still be used as an indicator of the students' activity or inactivity throughout the day.

As Figure 3(a) shows, the "Exercise" detected for both groups is very similar in amplitude and there is no significant difference between groups. However, we can see that the level for G2 is slightly lower than for G1 in the week of the exam. This could indicate that students from G2 in fact studied more and stayed more inactive due to that fact. For the "Still" classification, we can see from the Figure 3(b) that there is a difference between both groups. Students in group G2 tend to have a higher percentage of their time classified as "Still" when compared to the values from G1. However, this could result from different use of the smartphone between groups, i.e., the values from the classification only refer to moments where the user takes the smartphone with them. The fact that the classification only infers activity when students have the smartphone also explains the high percentage for both groups. Apart from that, most of the time that students spent at the University is to attend classes and, as such, they remain sitting down. Students from G2 could have a lower level of use of the smartphone and, as such, they have more time "Still". But, nonetheless, this represents a difference in their behavioral patterns and could have an impact in the students' performance.

For the label "In Vehicle", the classification takes in account movements in vehicles such as cars, bus and motorcycles. As depicted in Figure 3(c), in this classification there is no particular difference in the behaviors for both groups. Both seem to spend a low percentage of their daily time in vehicles, with values ranging from 36 minutes to 1 hour and 12 minutes in most cases. However, there are cases where values fall outside that time gap. One of those cases is the date before the exam for $\mathrm{G} 1$, where the value of time spent in vehicles, by students from G1, more than doubles. We are still uncertain of what could be the reason behind this. It represents nonetheless, a behavior changed when compared to G2. This also correlates with the theory that students from G2 stay more inactive before the exam in order to study.

Additionally, the "Unknown" classification also shows different patterns for both groups, as depicted in Figure 3(d). The "Unknown" classification is given when the classifier cannot identify any of the other possible classifications with enough precision. We believe that this data also translates a behavior pattern of smartphone use from the students, i.e., students from G1 use the smartphone more frequently and do drastic movements that generate the "Unknown" classification, while students from G2 seem to be calmer, while using the smartphone and maintain a very low and uniform value of "Unknown" classifications. 


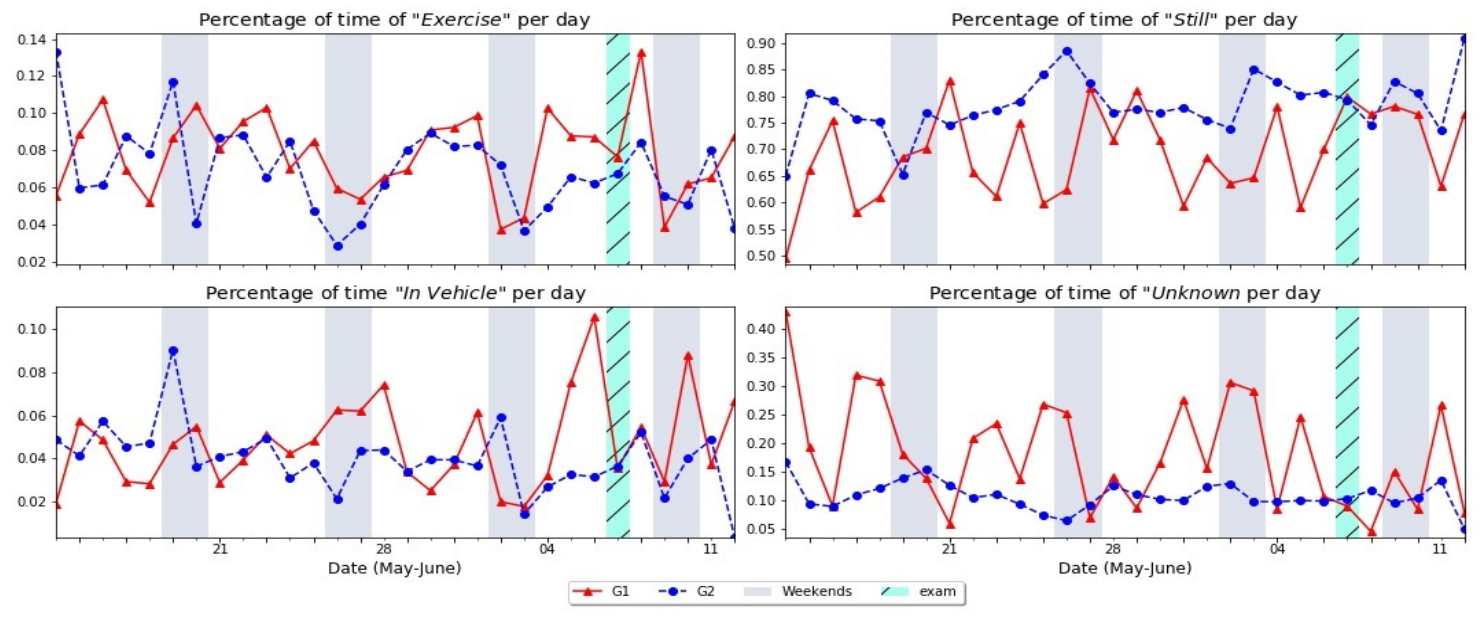

Figure 3: Percentage of time of "Exercise" per day (a); Percentage of time of "Still" per day(b); Percentage of time "In Vehicle" per day (c); Percentage of time of "Unknown" per day (d);
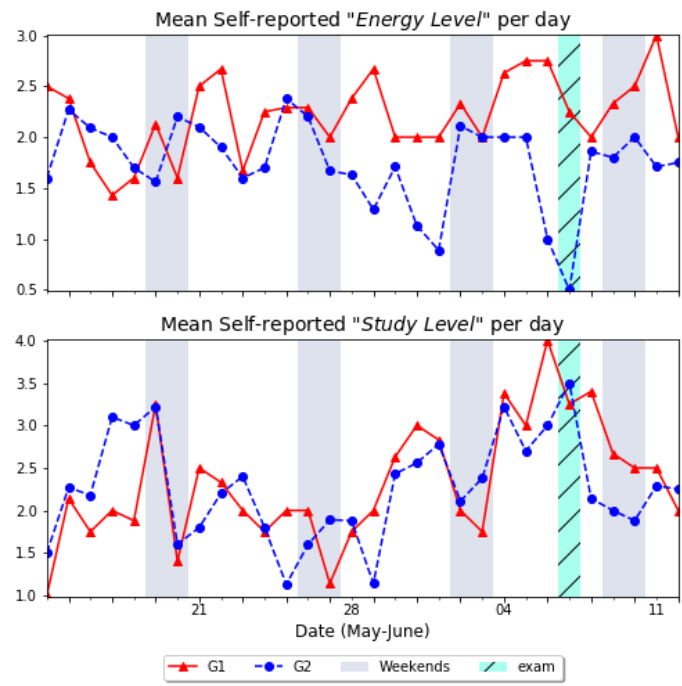

Figure 4: Mean value of Self-reported: 'Energy Level"(a); "Study Level" (b);

\subsection{Self-Reported data correlation results}

In this section, we present some of the results extracted from the Ecuador's dataset. More precisely, we compare the variables from the self-reported form (ground-truth) relation to each other. The self-reported data cover several aspects of the student physical, social and emotional states. The variables considered were: the study, the Sociability, the Quality of the Sleep, the "Energy" and the Number of Hours of Sleep. All these variables, except the Number of Sleeping hours, which were the real number, were evaluated in a scale of 0 to 4 inclusive.

The results in Figure 5 correspond to the evaluation of the progression of the students' "Energy" levels (a) in comparison to their "Study" levels (b). The values of the students' Study levels for both groups are in the bottom chart and the values for the "Energy" of both groups are in the top one. The values were obtained by collecting the answer of

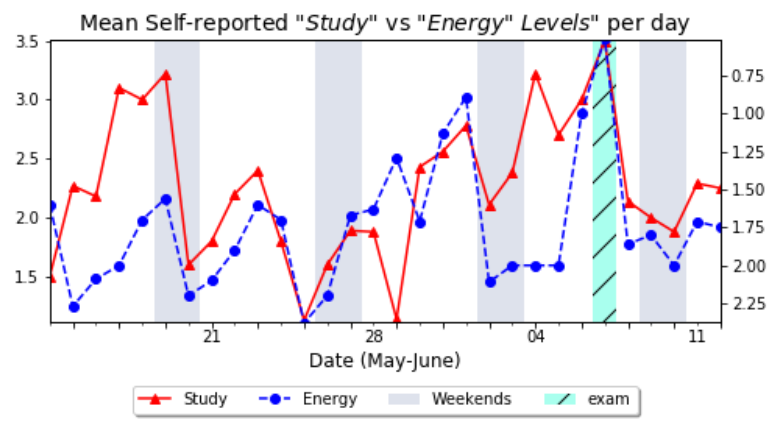

Figure 5: Mean Self-reported "Study" levels against the inverted Means value of Self-reported "Energy" levels per day, for students of G2.

the users to two daily questions, namely: "How do you feel today?" and "How much did you study yesterday?". As we can see in the figure, students from G2 have a more progressive evolution in their study levels and maximize their study level at the day of the exam. Students in G1 seem to have more drastic changes in their study levels, with a greater variance $(\sigma \mathrm{g} 1=0.69, \sigma \mathrm{g} 2=0.59)$ and decrease their study level the day before the exam.

Additionally, the patterns in the "energy" levels are different for both groups: students from G2 decrease their "energy" as they increase their study levels, while students from G1 maintain higher values of "Energy". These conclusions can also be drawn from the Pearson's correlation coefficient values $(\mathrm{RG} 1=0.328, \mathrm{RG} 2=-0.44)$. Concerning the "Energy" as well, students G1 have generally higher levels than students G2. This could in fact mean that students from G2 do in fact study harder than students from G1 and feel less "energetic" due to that.

To better visualize this correlation for students in G2, we add the graph in Figure 6. In this graph, we inverted the y-axis for the "energy" data, and we can see from the Figure 6 that curves align in several moments. We can assume that students from this group tend to feel less "energetic" as they 
increase their study time. The same did not happen for students in G1.

\section{CONCLUSIONS}

In this paper, we presented a HiTLCPS platform, dubbed ISABELA. In addition to actively involving humans as central actors in the system, ISABELA integrates several data sources, namely IoT data, smartphone-originated data, and Online Social Networks data. The platform was implemented using open-source IoT solutions, technologies, and standards, as well as interactive agents. The latter allows the system to perform context-aware actuation to the users, thus addressing one of the biggest open-issues in the state-of-the-art.

In order to test the platform, a trial was conducted, involving 30 students from Ecuador. This allowed to validate the functional aspects of the platform, namely the integration of the various sources of sensed data, including Online Social Networks data, and the inclusion of human users in the feedback loop.

Although the platform is fully operational, this was just a first implementation that opens up a whole range of new research avenues. In the near future, we will further explore HiTLCPS and the data gathered to create new models that automatically detect risk behaviors in failing students. New actuation techniques will also be addressed in future endeavors.

\section{ACKNOWLEDGMENTS}

The work presented in this paper was partially carried out in the scope of the SOCIALITE Project (PTDC/EEISCR/2072/2014) and the MOBIWISE Project (P2020 SAICTPAC/0011/2015), both co-financed by COMPETE 2020, Portugal 2020 - Operational Program for Competitiveness and Internationalization (POCI), European Union's ERDF (European Regional Development Fund) and the Portuguese Foundation for Science and Technology (FCT). Partial support was also given by Escuela Politécnica Nacional de Ecuador, and SENESCYT - Secretaría Nacional de Educación Superior, Ciencia, Tecnología e Innovacíon de Ecuador.

\section{REFERENCES}

[1] A. Kevin, "That' Internet of Things ' Thing," RFiD Journal, 2010.

[2] S. K. Khaitan and J. D. McCalley, "Design techniques and applications of cyberphysical systems: A survey," IEEE Systems Journal, 2015.

[3] D. Nunes, J. S. Silva, and F. Boavida, A Practical Introduction to Human-in-the-Loop Cyber-Physical Systems. Ltd., John Wiley \& Sons, 2018.

[4] D. S. Sousa Nunes, P. Zhang, and J. Sa Silva, "A Survey on human-in-The-loop applications towards an internet of all," IEEE Communications Surveys and Tutorials, 2015.

[5] N. Armando, A. Rodrigues, V. Pereira, J. Sá Silva, and F. Boavida, "An outlook on physical and virtual sensors for a socially interactive internet," Sensors, vol. 18, no. 8, p. 2578, 2018.

[6] J.-K. Min, A. Doryab, J. Wiese, S. Amini, J. Zimmerman, and J. I. Hong, "Toss 'n' turn," in Proceedings of the 32nd annual ACM conference on Human factors in computing systems - CHI '14, 2014.
[7] Z. Chen, M. Lin, F. Chen, N. Lane, G. Cardone, R. Wang, T. Li, Y. Chen, T. Choudhury, and A. Cambell, "Unobtrusive Sleep Monitoring using Smartphones," in Proceedings of the ICTs for improving Patients Rehabilitation Research Techniques, 2013.

[8] N. D. Lane, M. Mohammod, M. Lin, X. Yang, H. Lu, S. Ali, A. Doryab, E. Berke, T. Choudhury, and A. T. Campbell, "BeWell: A Smartphone Application to Monitor, Model and Promote Wellbeing," 5th international ICST conference on pervasive computing technologies for healthcare, 2011.

[9] R. Wang, F. Chen, Z. Chen, T. Li, G. Harari, S. Tignor, X. Zhou, D. Ben-Zeev, and A. T. Campbell, "StudentLife: assessing mental health, academic performance and behavioral trends of college students using smartphones," Proceedings of the 2014 ACM International Joint Conference on Pervasive and Ubiquitous Computing, pp. 3-14, 2014.

[10] A. Zenonos, A. Khan, G. Kalogridis, S. Vatsikas, T. Lewis, and M. Sooriyabandara, "HealthyOffice: Mood recognition at work using smartphones and wearable sensors," in 2016 IEEE International Conference on Pervasive Computing and Communication Workshops, PerCom Workshops 2016, 2016.

[11] M. Fazio, A. Celesti, F. G. Marquez, A. Glikson, and M. Villari, "Exploiting the FIWARE cloud platform to develop a remote patient monitoring system," Proc. IEEE Symp. Comput. Commun., vol. 2016-Febru, pp. 264-270, 2016.

[12] Telefonica, "IoTAgent Development Framework," https://github.com/telefonicaid/fiware-IoTAgentCplusplus, 2018-10-01.

[13] S. Krco, B. Pokric, and F. Carrez, "Designing IoT architecture(s): A European perspective," 2014 IEEE World Forum Internet Things, WF-IoT 2014, pp. 79-84, 2014.

[14] Telefonica, "Fiware Cygnus," https://github.com/telefonicaid/fiware-cygnus, 21Jan- 2018.

[15] Telefonica, "FIWARE Short Time Historic," https://github.com/telefonicaid/fiware-cygnus 21Jan- 2018.

[16] Grupo de Internet de Nueva Generación, "Identity Manager - Keyrock," https://github.com/ging/fiware-idm, 21- Jan- 2018.

[17] R. Milton, D. Hay, S. Gray, B. Buyuklieva, and A. Hudson-Smith, "Smart IoT and Soft AI," in Living Internet Things Cybersecurity IoT - 2018, pp. 1-6, 2018.

[18] D. Wang, B. K. Szymanski, T. Abdelzaher, H. Ji, and L. Kaplan, "The Age of Social Sensing," arXiv preprint arXiv:1801.09116, 2018.

[19] J.Fernandes, D.Raposo, N.Armando, S.Sinche, J.Sá Silva, A.Rodrigues, V.Pereira and F.Boavida, "An Integrated Approach to Human-in-the-Loop Systems and Online Social Sensing," IEEE CAOS19, Manuscript submitted for publication., 2019. 